\title{
Introduction à une étude du sacrifice chez les
} Bobo de Haute-Volta

Introduction to a study of sacrifices among the Bobo of Upper Volta

\section{Guy Le Moal}

\section{(2) OpenEdition}

1 Journals

Édition électronique

URL : http://journals.openedition.org/span/528

DOI : $10.4000 /$ span. 528

ISSN : 2268-1558

Éditeur

École pratique des hautes études. Sciences humaines

\section{Édition imprimée}

Date de publication : 30 mai 1981

Pagination : 99-126

ISSN : 0294-7080

\section{Référence électronique}

Guy Le Moal, «Introduction à une étude du sacrifice chez les Bobo de Haute-Volta », Systèmes de pensée en Afrique noire [En ligne], 5 | 1981, mis en ligne le 05 juin 2013, consulté le 19 avril 2019. URL : http://journals.openedition.org/span/528; DOI : 10.4000/span.528 


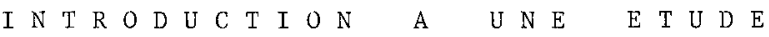

DU SACR I F I C E C H E Z L E S B O B O
DE H A U T E V O L T A
par

Guy Le roal

Sacrifier, chez les Bobo comme dans la plupart des populations d'Afrique noire, est un acte si fréquent, si quotidiennement rêpété - si banal donc - qu'en dépit de ses modalités infiniment variées et de ses implications de toute évidence riches de sens, il tend paradoxalement à échapper à $1^{\prime}$ attention et à n'être que rarement reconnu comme un sujet d'étude en soi.

Pourtant, par sa quasi universalité, le sacrifice apparait à l'esprit de beaucoup comme l'acte religieux par excellence et le seul peutêtre exclusif de ce système de croyances.

Etudier le sacrifice dans une société donnée ne peut être qu'une entreprise d'envergure puisque par lui c'est à tous les paliers de 1 'institution religieuse qu'on accède. Privilégier tel ou tel aspect du sacrifice risque donc d'en amoindrir voire d'en déformer le sens. Seule, nous a-t-il semblé, une approche volontairement globale et fondée sur un recueil systêmatique et même, dans la limite du possible, exhaustif de données précises peut rendre compte objectivement de cet ensemble complexe de rituels qu'est le sacrifice.

Le village étant, chez les Bobo, une catégorie sociale pertinente, c'est à ce niveau que s'est développée notre enquête. Choisissant donc un village-type (Kouroumani, arrondissement de Solenzo), nous avons fait le recensement exhaustif des lieux sacrés (128) et étudié dans le détail les modalités des sacrifices qui se déroulent sur les 61 autels voués à des cultes actifs.

De cette enquête et de ses rêsultats (portant essentiellement sur 
l'énoncē et le dêchiffrement d'un code des victimes sacrificielles en rapport avec les diverses catégories spirituelles bobo), il ne sera que partiellement question ici. Il nous a en effet paru nécessaire de donner en préalable quelques indications sur le lexique bobo du sacrifice et surtout de décrire et de commenter le dêroulement habituel des rites sacrificiels.

\section{Le Zexique}

Pour traduire ce que nous désignons sous le nom très gēnéral et à vrai dire très vague de "sacrifice", les Bobo ne semblent pas disposer d'un terme vraiment spécifique; en revanche, à côté de mots d'usage courant, ils emploient des termes spécialisês pour certains actes rituels tel que l'immolation.

Le mot le plus ordinairement utilisé par les Bobo, s'agissant du sacrifice considéré de façon très globale, est tyere. tyere est un substantif composé, il est formé d'un verbe, tye ou tè, et de la particule ta, pluriel re.

tye signifie "faire" ou plus exactement "se faire" dans le sens de "devenir", "commencer à être", il est employé pour traduire des expressions comme "il se fait vieux" (i.e. "il est en train de devenir vieux") ou "la bière est en train de se faire". tye est à distinguer de ta "faire" qui est plus factitif et a le sens courant "d'agir", "accomplir". Il y a là une nuance qui n'est sûrement pas gratuite et qui mériterait d'être êlucidêe.

ta, pl. re, finale d'agent, sert à désigner celui qui agit et semble ici souligner que c'est le locuteur qui est 1'auteur de l'action qui "se fait". tyere, c'est en quelque sorte "le fait d'être en train de faire..." mais 1 'objet de 1 'action reste non exprimé et seulement sous-entendu. En général, lorsqu'on dit a yo tyere na, on comprend "je vais faire"... sous-entendu : un sacrifice. Mais, comme 1'expression peut éventuellement faire allusion à une action quelconque un travail, une promenade - on peut, en redoublant le terme dissiper toute équivoque : on dira a yo tyere - tyere et chacun comprendra que c'est bien un sacrifice que l'on va faire. Ainsi tyere est une façon commode de désigner de façon très générale tout ce qui a trait au sa- 
crifice sans avoir recours à un terme spécifique trop révélateur.

Quoique beaucoup plus précis, un autre terme reste, lui aussi, allusif : c'est yare, qui désigne I'action de "tuer". yare, qui ne peut évidemment être employê que dans le cas de sacrifice sanglant, s'accompagne toujours de la mention de l'animal, le plus souvent un poulet car yare, de surcroît, ne s'applique guère qu'à de petits sacrifices sanglants. On dit yo nan' yare "aller tuer (sous-entendu sacrifier) une poule".

Lorsqu'il s'agit de sacrifices plus importants, comportant la mise à mort de quadrupèdes, on utilise un terme plus intéressant car il possède le sens, cette fois tout à fait spécifique, d"'immoler" : c'est kêñre (de kè).

Un autre terme existe, lui aussi propre au lexique sacrificiel, qui ne s'applique qu'à des sacrifices très particuliers destinés à obtenir de certaines entitês précises une réponse à une question posée sur un sujet généralement grave (il ne s'agit pas d'un sacrifice divinatoire bana1). En fait, bèn, le terme en question, n'est employé que Iorsqu'on égorge un poulet sur les autels de sogo ou soppè (1) afin de connaître les raisons d'un événement grave (une mort soudaine et suspecte par exemple).

Le vocabulaire du don n'offre, de son côté, aucun terme exclusif de l'emploi courant. Tout ce qui est apporté sur les autels, depuis l'eau des libations jusqu'aux animaux de sacrifice est dit simplement être "donné" : $k \alpha$ ou pere. On peut aussi employer $b i$, un terme d'usage courant mais qui, dans le contexte sacrificiel, prend une signification

(1) Rappelons que les principales entités spirituelles bobo sont : wuro, le Dieu suprême; kwere, la Foudre; zo, l'Eau; sogo, la Brousse; dwo, émanation de wuro qui se manifeste sous la forme du masque; funanyone, les "Esprits"; wiyage, les "Génies" et enfin les Ancêtres (deux autels : borè et saprè) auxquels se rattache kiri, le village en tant que concept spirituel. Pour plus de précisions sur ces termes et sur ceux qui apparaissent dans cet article on pourra se reporter à : Le Moa1, G., Les 3 obo. Nature et fonction des Masques, Paris, Travaux et Documents de $1^{\prime}$ O.R.S.T.O.M. $\mathrm{n}^{\circ} 121,1980, \mathrm{X}+535 \mathrm{p} ., 37$ fig., 36 photos, 3 cartes H.T., ind., biblio. Dans le présent texte, les références à cet ouvrage se présentent sous la forme abrégée suivante : (G.L.M., $1980, p(.$.$) .).$ 
plus riche ét éclaire un peu sur la destination supposée des biens offerts. bi, en effet, veut dire "prendre", ainsi, par exemple, dénomme-t-on les offrandes de bière sur 1 'autel $k \hat{u} i$ bi dâga; mais $b i$ contient aussi 1 'idée que la chose prise est déplacée, emportée ailleurs. Ainsi se révéle, par le vocabulaire, le fait que, pour les Bobo, la bière et les autres offrandes, comme d'ailleurs le sang versé, ne sont pas "consommés" sur place mais pris et emportés jusqu'au lieu de leur sêjour par les entités spirituelles destinataires.

Au total, il semble que deux mots seulement, kè et bèn, soient réservés à un emploi dans le cadre exclusif du sacrifice. Or, dans les deux cas, il s'agit de mise à mort d'animaux. Cela tendrait à accréditer 1 'idêe - qui ressort tant de nos propres investigations que des opinions souvent formulées par les Bobo eux-mêmes - que l'immolation est l'acte majeur et en quelque sorte distinctif de ce que d'une façon très générale on appelle "le sacrifice".

Moment culminant du sacrifice, la mise à mort n'est pourtant que 1 'un des très nombreux rituels qui ponctuent le déroulement d'une pratique religieuse qui se signale par son caractère fortement totaIisant.

Dans un premier temps, c'est donc à la description et à l'analyse de toutes les phases successives du procès sacrificiel que nous limiterons notre propos.

II. Les phases du procès sacrificiel

1. Le dépôt de za cendre.

Le procès sacrificiel, quelles que soient les formes qu'il prendra et quelles que soient ses raisons et ses fins, est obligatoirement soumis à une formalité rituelle préalable : le yibe tege dâga "le dépôt (tege) de la cendre (yibe)".

Lorsque la décision a été prise de faire un sacrifice, le sacrifiant se rend sur $1^{\prime}$ autel de l'entité spirituelle concernêe. Sur la plupart des autels mineurs le sacrifiant agit de son propre chef et peut même opérer solitairement, pour nombre d'autels importants, tels que borè, saprè ou dwosa la présence d'un chef de lignage pleinement 
investi (le sapro), ou celle d'un responsable du culte (le dwobwo pour le culte de cho), ou encore celle d'un koronate (alliê à plaisanterie jouant le rôle institutionnel de médiateur-cf. infra) est requise.

Accroupi devant 1'autel, le sacrifiant commence un long discours qui comporte d'abord un "exposé des motifs" : genèse des faits et description du cas avec toutes les précisions utiles. Vient ensuite 1 'énoncé des requêtes, c'est à dire la formulation sans détours de ce que $1^{\prime}$ on attend : pitiê, pardon, santé, enfants, récoltes... biens dont la liste est évidemment sans fin, mais dont le choix doit rester en rapport avec les compétences personnelles de 1 'entité devant 1 'autel de laquelle on se présente. Enfin, pour terminer, sont décrits avec précision les dons qui seront faits en retour : animaux qui seront sacrifiës, actions expiatoires diverses.

Si la présence d'un tiers est nécessaire, sapro ou koronate, son rôle se borne à répéter mot pour mot le discours du sacrifiant en l'agrémentant au besoin de commentaires approbateurs.

Ce premier et nécessaire contact par le Verbe est suivi de 1 'acte rituel par lequel va se clore cette courte et simple cérémonie : le sacrifiant, ou l'officiant désigné comme intermédiaire, "pose" (tege), ou plus exactement déverse la cendre contenue dans une calebasse-louche qu'il tient de sa main droite sur l'autel même.

A ce stade prêliminaire du procès sacrificiel, le but poursuivi est essentiellement de créer une situation "contractuelle". La problèmatique sacrificielle met en avant la présupposition que le sang versé, les offrandes déposées, les prières même, font nâ̂tre une obligation de réponse (fût-elle négative) chez le destinataire sacré. Cet aspect "contractuel" du sacrifice a bien été relevé par certains auteurs, notamment par E. Durkheim - comme le rappelait ici même J.F. Vincent (1) - mais sans doute n'a-t-il pas été suffisamment pris en compte.

(1). "Le sacrifice chez les Mofu". Systèmes de pensée en Afrique noire, cahier 2, 1976, p. 198. 
Chez les Bobo, donc, existe clairement I'expression d'un contrat dans la pratique sacrificielle, mais l'originalité de la démarche bobo réside dans le fait que ce n'est pas par le sacrifice lui-même, c'est à dire par les dons de denrées végêtales ou de victimes animales, que se ratifie le contrat en question : c'est préalablement, lors du "dépôt de la cendre", que s'engagent effectivement les parties.

Le yibe tege dâga une fois accompli, on attend de l'entité propitiée qu'elle agisse et ce n'est que lorsque cette dernière a exaucé ses voeux que le demandeur, cette fois devenu dëbiteur, devra enfin effectuer le sacrifice promis.

En somme, avec un pragmatisme évident, on laisse le soin aux entités spirituelles de remplir les premières leurs obligations; ce serait pourtant se tromper lourdement qu'imaginer dans l'esprit des Bobo une possible intention de se dérober une fois le bienfait acquis. En effet, le sacrificateur, au moment du yibe tege dâga, a apporté une caution qui 1 'engage irrévocablement, une caution de qualité si reconnue qu'elle peut rassurer le créancier sacré et justifier d'ailleurs pleinement les plus terribles représailles de sa part en cas d'inexécution des promesses faites.

L'instrument de cette caution, c'est la cendre.

La cendre est une matière hautement valorisée chez les Bobo et employée a des fins religieuses aussi nombreuses que diverses (G.L.M. 1980, index p. 519).

De la cendre, tout d'abord, on dit qu'elle est indélébile; sa trace, même imperceptible, demeure : elle est donc tout à fait appropriée pour symboliser l'imprescribilité d'une dette. Mais la cendre n'est pas employée seulement pour ce pouvoir évocateur, elle est considérée comme un substitut réel, elle préfigure matériellement et en toute équivalence ce qui a été promis; en français, les Bobo qui connaissent nos usages commerciaux disent : "La cendre, c'est comme un bon que 1 'on signe". Dans ces conditions, on comprend que la cendre ait pleine valeur de caution et qu'on puisse même, dans une certaine mesure, considérer son dépôt comme un véritable sacrifice par anticipation. Concernant la cendre, il y a plus encore. "La cendre, précisent les Bobo, est le koronate des ancêtres" (yibe we guimikôma koronate), ce qui signifie que la cendre joue à 1 'égard des ancêtres (et des 
autres entités spirituelles d'ailleurs) le même rôle que le koronate (1) : c'est dire qu'elle est un intermédiaire, un agent de médiation entre les deux parties contractantes, mais c'est dire aussi et peut-être surtout qu'elle est un puissant moyen d'action pour qui en fait usage à son profit. Pour comprendre de quel poids sont les fonctions du koronate et, partant, de la cendre puisqu'on 1 'en rapproche, il faut savoir en effet que le koronate, lorsqu'il est fait appel à lui, ne se contente pas d'un simple arbitrage : son acte est doté de la force exécutoire - nu1 ne peut refuser au koronate l'accord qu'il prône ou la réconciliation qu'il conseille. De la même façon, pense-t-on, en versant la cendre sur l'autel on fait un acte d'où découlent des obligations impératives pour les deux parties : l'entité spirituelle concernée ne peut se dérober à la démarche du sacrificateur opérant sous les espèces de la cendre et même elle est contrainte de répondre à sa demande, en retour de quoi, elle est assurêe de recevoir le sacrifice puisque, nous 1 'avons vu, cette même cendre en est la caution.

La cendre, il faut enfin le signaler, a aussi une place significative dans le registre des représentations abstraites en rapport avec le sacrifice.

La symbolique bobo fait grand usage d'un système d'oppositions conceptuelles duelles parmi lesquelles revient très souvent le couple Sec/Humide qui, par un enchaînement dialectique, mène aux grandes catégories dualistes classiques Nature/Culture en passant par : Chaud/Froid, Danger/Sécurité, Brousse/Village etc... La cendre, naturellement, est la parfaite image de la siccité; dans l'ordre du Sec, cependant, sa consistance lui donne les mêmes caractères physiques que les liquides : la cendre "coule" comme l'eau, comme le sang, comme la sève, comme ces fluides qui sont par excellence,

(1). Le koronate, "maître du pardon (koro)", est un allié à plaisanterie dont la fonction essentielle est de résoudre les conflits intervenant entre deux individus d'un même lignage (G.L.M., 1980, p. 351). 
chez les Bobo, les vecteurs de forces vitales ou les réceptacles d'entitês spirituelles. Ainsi la cendre présente-t-elle l'avantage d'être un équivalent des matières sacrificielles liquides tout en appartenant, sur le plan conceptuel, à la catégorie qui leur est opposée.

Cet usage des qualités particulières de la cendre procède de la nécessité, dans un tel système, d'assurer un juste équilibre des contraires au moment où vont devoir s'opérer les transferts de forces déclenchés par le procès sacrificiel.

Après le yibe tege dâga, s'écoule un temps plus ou moins long. Lorsqu'il s'agit, de la part du sacrifiant, d'une demande expresse, qu'elle soit personnelle ou faite au nom d'une collectivité, nous avons dit qu'on attend d'être exaucé pour faire le sacrifice. Lorsqu'il s'agit d'un sacrifice périodique, commandé par la pratique ordinaire d'un culte ou la mise en oeuvre d'un cérémonial institutionne1 (rites de passage, rites agraires, etc.), le dêpôt de la cendre a également lieu et il a le même caractère contractuel, mais une réponse n'étant pas attendue à ce stade, la date du sacrifice est fixée en fonction de diverses opportunités matérielles et spirituelles.

2. Préliminaires du sacrifice.

C'est à l'initiateur du sacrifice - au "sacrifiant" - que revient la responsabilité d'en organiser la préparation matérielle et notamment de programmer soigneusement les phases de la fabrication de la bière - ingrédient nécessaire - de telle sorte que celle ci soit prête au jour dit.

Pendant cette période de brassage de la bière s'établit une série de rapports entre le sacrifiant et l'entitê destinataire du sacrifice - par prêtres interposés si besoin est. Avant même, par exemple, que commencent les opérations, dès qu'a été prise la décision de faire le sacrifice et choisie sa date précise, le sacrifiant se doit d'en informer l'entité concernée en s'adressant à elle, personnellement ou par le truchement d'un prêtre, devant l'autel. Ce petit rituel d'avertissement, purement oral, a pour effet de mettre en action tout un processus de relations préétablies entre les hommes et 
la hiêrarchie des entitês.

Nous avons montré ailleurs (1) comment se réalise cette communication entre les hommes et le monde des entités spirituelles et par quel circuit les paroles des prières aussi bien que l'essence des matières sacrificielles (sang, denrẹes oblatives) atteignent 1a plus haute instance, wuro, pour que s'exprime, en retour, l'intention divine.

Au stade actuel du procès sacrificiel, aucune réponse n'est encore sollicitée et il s'agit seulement d'instruire la ou les entités intéressées de 1'état d'avancement des opérations de brassage de la bière

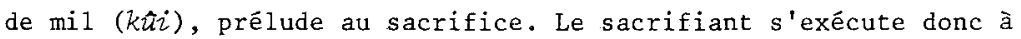
plusieurs reprises. Tout d'abord, au cours du maltage, lorsque, dûment mouillés, les grains ont germé (6è jour en principe); en second lieu, peu après (8è jour), lorsque le malt est sec; enfin, ultime démarche, lorsqu'après le brassage proprement dit une première ébullition a fourni cette décoction qu'on appelle kîui fu.

Un peu de kâi fu sera déposé sur l'autel, message clair pour tous et qui indique que $1 a$ bière sera "mûre". (kûi pra) le surlendemain et qu'alors aura lieu le sacrifice.

\section{Paroles.}

Tout sacrifice commence par des discours. Le sacrifiant en premier, puis le sacrificateur (ou, si le sacrifiant est également sacrificateur, une personne désignée pour l'office liturgique d'orant) reprennent les discours et les prières qui avaient été dits devant l'autel lors du rituel préalable du "dépôt de la cendre".

\section{Libations.}

La phrase consacrée à l'oralité étant achevée, l'action est inaugurée par une série de libations.

(1). G.L.M., 1980, p. 155-159 et fig. 5, 6. 
Tout d'abord c'est à nouveau de 1a cendre que 1 'on dépose sur 1'autel en prononçant une courte prière. Cela ne souffre pas d'exception : la cendre est acceptée par toutes les entités spirituelles: La cendre va jouer ici son rôle de médiation et introduire aux actes qui vont suivre; de plus, par sa seule présence, elle va rappeler le "contrat" qu'elle avait servi à sceller et témoigner de son imminente exécution. On notera également au passage que c'est conformẻment à sa nature de "liquide sec" qu'elle est employée ici à titre de libation et cela conjointement avec de l'eau ou de la bière.

Aussitôt après la cendre, ce sont en effet des corps essentiellement aqueux qui sont versés sur 1 'autel et cela, dit-on couramment, pour "nettoyer" ou encore "mouiller la bouche" des ancêtres ou des entitës destinataires diverses.

A ce propos, il nous fall attirer 1 'attention sur le fait que la nature du liquide répandu est étroitement déterminée par l'identité du destinataire sacré. C'est ici.qu'apparâ̂t la première trace probante de l'existence d'un code sacrificiel cotant les différences existant entre les grandes catëgories spirituelles auxquelles se rattachent toutes les entités et permettant d'étudier le jeu complexe de leurs rapports mutuels.

Dans le cadre du présent article; nous nous bornerons à indiquer quelles sont les libations (et les nourritures oblatives) parmi lesquelles les diverses entités spirituelles peuvent exercer leur choix.

a) - La bière de mil.

A une seule exception près (une certaine figure de dwo appelée Kwele dwo qui est réfractaire à tous les liquides aqueux), tous les autels reçoivent des libations de bière. En fait, ici comme dans nombre de populations africaines, la bière est inséparable de 1'acte sacrificiel, de telle sorte qu'il n'est pas de cêlébration religieuse sans préparation de bière.

b) - L'eau.

I1 convient de bien distinguer entre 1 'eau pure et l'eau entrant en composition avec de la farine. 
- L'eau pure, est une libation exclusivement réservêe aux autels d'ancêtres (borè).

- L'eau mise en composition avec de la farine donne, selon le mode de préparation deux produits très différents appelés respectivement sêkèlè et fogo 30 .

- sêkètè est en fait une sorte de pâte de mil non cuite. sêkèzè est obtenu à partir de "petit mil" (Pennisetron, dugo furu en bobo), denrée éminemment sacrée puisqu'elle fût la première nourriture donnée aux hommes aux temps cosmogoniques par le Dieu suprême wuro en personne. Ce petit mil est pilé au mortier, puis lavé et pilè à nouveau alors qu'il est encore humide. Cela donne une pâte assez ferme à laquelle on ajoute du sel (mais jamais de piment). Très vite de l'eau apparaît au dessus de la pâte et c'est cette eau un peu trouble qui est versée sur 1'autel. Quant à la pâte de sêkèzè, elle constitue la seule nourriture oblative qu'emploient les Bobo et c'est plus tard, après les sacrifices sanglants, qu'une parcelle en sera placée sur l'autel en prêlude à un repas où le sêkèzè restant accompagnera la chair des animaux sacrifiés.

- fogo zo est seulement constitué par un peu de farine délayée dans de l'eau, sans la moindre trace de sel ou piment. La farine de petit mil peut être employée, mais ce n'est pas obligatoire et $I^{\prime}$ on peut utiliser du sorgho blanc, denrée sans valeur religieuse particuliẽre. Ouant au maís, il est tout à fait exclu. Il ne s'agit pas, avec fogo zo, d'une véritable nourriture, personne d'ailleurs n'en absorbe au cours du rite, mais d'une matière seulement réservêe aux libations.

A considérer ces deux derniers produits, il se dégage l'impression que 1 'un est plus valorisé que l'autre et que de leur emploi dépend la plus ou moins grande solennité de l'acte sacrificiel. Il est certain que le sacrifice au cours duquel il est fait usage de sêkèzè suppose 1 'engagement de plus grandes dëpenses et un plus haut degré de participation. D'une part, l'emploi de sêkèzè entraîne obligatoirement le choix de la victime sacrificielle la plus marquante - un mouton par- 
faitement blanc; d'autre part la préparation du sêkèle demande des quantitês relativement importantes d'une denrée de prix, le petit mil, dont on connait le caractère sacré et qui est en outre un bien de nature communautaire (foroba). Cette préparation, par ailleurs, requiert la collaboration des femmes (façon peut-être de les introduire dans un acte où elles ont peu de place). Enfin et surtout, l'offrande de sêkèzè s'accompagne nécessairement d'un repas qui peut rassembler un nombre important de personnes et qui, s'il n'est peut-être pas un acte "communiel" au plein sens religieux du mot, est tout au moins un acte d'union vivement ressenti aussi bien sur le plan spirituel que social ainsi qu'en témoigne 1 'atmosphère régnante, faite à la fois de ferveur et de familiaritê joyeuse.

En comparaison, le rite sacrificiel où seul est utilisé fogo zo semble plus simple. La farine est délayée sur place en quantité juste suffisante pour mouiller un peu l'autelet, surtout, il n'y a pas partage entre les participants et repas collectif.

En dépit de ces apparences, il faut se garder de croire que peuvent correspondre aux deux types d'offrandes - sêkèzè et fogo zo - deux catégories de sacrifices qualitativement inégales : des "grands" sacrifices à sêkèlè qu'on imaginerait volontiers réservés aux autels importants, de "petits" sacrifices à fogo zo pour les autels mineurs.

A cet êgard, quatre faits sont à prendre en considération.

- Plusieurs entités spirituelles, et non des moindres, refusent toute libation ou nourriture à base de farine. dwo, par exemple, comme 1'indique ce précepte : dwo mâna fogo di, ti ma na yibe ("dwo refuse la farine, c'est la cendre qu'il faut lui apporter").

- A 1 'inverse, dans un cas au moins (sur 1 'enclume tokwiyanô), les deux types de libations doivent être effectués conjointement.

- De toutes façons, même lorsque I'usage de sêkèlè est prescrit, une certaine latitude existe qui permet, en cas d'urgence par exemple, de substituer du fogo zo.

- Enfin, sans avoir même à procéder à ùne enquête exhaustive, il suffit de voir, par exemple, verser du sêkèzè sur l'autel du Dieu suprême wuro pour comprendre que de la seule observation 
du type d'offrande pratiqué on ne peut inférer de la qualité d'une entité et de sa place dans une éventuelle hiérarchie.

Si 1 'existence d'un code se profile bien derrière ces règles d'emploi des diverses offrandes, il est apparent que ce code met en jeu plusieurs autres variables. Nous allons d'ailleurs en avoir la preuve sans tarder.

Revenons au déroulement du sacrifice. Après le dépôt de la cendre, le sacrificateur procède donc aux libations. L'eau d'abord eau pure ou eau de sêkèzè ou encore fogo zo suivant le destinataire - puis la bière. Chaque libation est accompagnée d'une prière dont les paroles nous renseignent sur le sens vêritable que cet acte a aux yeux des Bobo.

Les simples libations d'eau ou de bière et même les offrandes de sêkèzè ne comptent pas normalement au nombre des dons qui sont promis aux entités en retour du bien qu'on attend d'elles. Encore qu'existe chez les Bobo des rituels où seules sont faites des offrannon sanglantes, il semble que le "vrai" sacrifice exige le don de vies animales - avec ou non versement de sang d'ailleurs. Des libations, certes, accompagnent nécessairement tout sacrifice sanglant, mais leur but est tout autre que celui qui a motivé le rite. L'objet principal du sacrifice, c'est à dire son but précis, n'est en effet pas évoqué au moment des libations. Les paroles qui sont dites alors montrent que le versement d'eau et de bière est un simple geste de civilité et un acte symbolique seulement propre à satisfaire l'intérêt général, à donner la "santē" à tous. En versant l'eau, le sacrificateur explique en effet que "Si quelqu'un veut parler à son père (et donc à ses ancêtres, comme à toute autre entité spirituelle) le matin, il lui donne de I'eau pour nettoyer sa bouche" et il ajoute : "Voilà l'eau froide, que la santé soit sur la maison" (1).

Les libations sont toujours faites avec une écuelle tenue de la main droite. Un peu de liquide est versè à terre, sur la droite

(1). sô za tire kwe ne tô urwwa - zo pra ma a do di sosogo ka ba zo kona, ma kona kô ka vye. 
de 1'autel; le restant est versé sur 1 'autel même. Pendant tout le temps de l'opération, le sacrificateur tient déjà dans sa main gauche le poulet qui sera sacrifié.

5. Immolation des victimes.

Les libations achevées, le sacrificateur fait passer le poulet dans sa main droite et commence aussitôt une prière dans laquelle, cette fois, il énonce avec précision les motifs rêels du sacrifice. C'est le rappel, un peu abrégé, de 1 '"exposé des motifs" fait dêjà par deux fois : à l'époque, d'abord, du dépôt de la cendre et, tout récemment, au début du présent rituel.

En conclusion de sa prière, le sacrificateur présente enfin le premier animal choisi pour être tué : "Voilà la poule, dit-il par exemple, je la donne à ..." (1) et il prononce le nom du destinataire sacrë.

Plus strictement encore que les produits destinés aux libations, les animaux voués aux sacrifices sont choisis selon un code subtil établi en fonction des prédilections personnelles attribuëes à chaque entité spirituelle. Ce code contient une liste limitée d'espèces animales pour chacune desquelles sont retenues un certain nombre de caractéristiques physiques : sexe, couleur, nature du plumage ou du pelage. Certaines modalités pratiques telles que la façon de capturer 1 'animal ou la technique de sa mise à mort pourront occasionnellement être prises aussi en considération.

a) - Les victimes sacrificielles.

- Les poulets.

En tête des espèces sacrifiables vient évidement le poulet, coq ou poule selon le cas et parfois même poussin. Tout sacrifice Bobo comporte obligatoirement l'immolation de poulets.

(1). ka ba nane, a pere... ma. 
Pour des sacrifices faits en des occasions mineures, on peut se contenter de tuer un seul poulet, mais le plus souvent deux poulets au moins sont nécessaires. Dans les grandes circonstances la plupart des entités spirituelles se voient sacrifier un quadrupède. En cé cas, on commence d'abord par sacrifier un premier poulet, "pour la santé des participants". Un second poulet est alors sacrifié; il précéde, ou plutôt dit-on - il "accompagne" le quadrupède : ce sont ces deux animaux qui vont constituer la part personnelle de l'entité spirituelle destinataire; ce sont eux qui représentent la dette contractée par le sacrifiant et leur mise à mort marque le moment le plus important du rite sacrificiel. Après la mort du quadrupède, un troisième pouletest alors sacrifié, à nouveau pour "la bonne santê". S'il s'agit d'une célébration collective, chacun des groupes participant fait alors tuer en son nom un ou plusieurs poulets. On peut assister ainsi à de véritables hécatombes.

La couleur du plumage est un important facteur pour le choix des poulets. Trois couleurs sont retenues : blanc, rouge, noir, mais une couleur précise peut aussi ne pas être exigée. L'affirmation, par les responsables d'un culte, que la couleur du poulet est "indifférente" doit être interprétée comme une prescription aussi impérative que celle d'une couleur donnée; en fait, sous la dénomination "couleur indifférente" on trouve aussi souvent des poulets au plumage bicolore que des poulets de l'une des trois couleurs citées ci-dessus. De toutes façons, étant expréssement choisie par certaines entités (la Brousse notamment), la prescription "couleur indifférente" constitue bien une catégorie en soi.

La nature du plumage ou certaines particularités physiques peuvent faire retenir prëférentiellement certains poulets. Parmi ces poulets hors du commun on peut citer :

- Le poulet baba, qui se distingue par des plumes courtes aux barbes fripées, chichement réparties sur le corps et pratiquement absentes sur les ailes. Ce volatile, qui a I'air d'être naturellement à demi-déplumé, jouit d'un 
grand prestige car il a sa place dans la mythologie, ayant étè - aux dires de certains - le premier oiseau créé.

Le baba n'est sacrifié qu'assez exceptionnellement et souvent d'une façon horrible puisqu'il est écartelé vif.

- Le poulet degeseni dont les plumes blanches "dêchirées" lui donnent I'apparence d'avoir une toison de mouton, ce qui lui vaut d'être, dans le code, l'équivalent d'un ovin.

- Le poulet bini nane, "poulet du mois de bini", dont les plumes sont de couleur indéfinissable - ni vraiment rouges ni vraiment blanches - comme le sont, dans la gamme des verts, les feuilles au mois de bini (septembre). Ce poulet entre dans la catégorie "couleur indifférente" comme le suivant, bicolore aussi, mais de façon plus tranchée.

- Le poulet gwenderi, dont la disposition des plumes rappelle celle des cauris (gwene) entassés, mais dont surtout le plumage est rayé soit rouge et blanc (genderi pene) soit noir et blanc (guendemi gîu).

- Le poulet kaba ma pene, dont la naissance des ailes (kaba) est rouge (pene), mais tout le reste du corps blanc. Ce poulet, bien que bicolore lui aussi, n'entre pas seulement dans la catégorie "couleur indifférente", mais peut être aussi apprécié d'entités qui privilégient soit le rouge, soit le blanc.

- Le poulet byê ton̂o (1itt. "cornes" - i.e. excroissances - "beaucoup") dont la crête présente une multitude de petites excroissances qui évoquent un monticule de graines de mil. A cause de cette particularité, ce poulet est très recherché pour les sacrifices agraires, en particulier on l'immole sur la terre même du champ avant la mise en culture dans l'espoir d'une bonne germination et avant 1 'épiaison pour obtenir une abondante récolte.

Dans le moment culminant des grandes cérémonies sacrificielles, 
avons-nous dit, s'ajoute à l'immolation de poulets celle de quadrupèdes. Ces animaux appartiennent à quatre espèces principales : ovins, caprins, bovins et chiens.

- Les ovins.

Les moutons - béliers ou brebis - sont des victimes sacrificielles de tout premier rang. En aucun cas, le mouton n'est utilisé comne animal de boucherie, il est exclusivement réservé aux sacrifices et sa chair n'est donc consommée qu'en cette circonstance.

L'élevage du mouton est le privilège absolu du chef de lignage (watô) mais à la condition qu'il ait reçu son investiture religieuse, c'est à dire qu'il soit pleinement apte aux fonctions de prêtrise (il porte dès lors le nom de sapro, pl. sapra). C'est le sapro donc qui gère le troupeau et décide seul de sacrifier tel ou tel mouton au nom de la collectivitê lignagère qu'il reprêsente. C'est aussi le sapro et lui seul qui égorge les moutons sur l'autel.

Si l'un des membres du lignage désire faire sacrifier un mouton à titre personnel, il doit, bien entendu, avoir l'accord du sappo et s'en remettre à lui comme sacrificateur, mais il doit, en outre, payer l'animal. Le sapro ne peut garder la somme reçue, il doit 1 a dépenser sans retard et, en principe, pour un achat utile à tous.

Une personne quelconque du lignage peut souhaiter se charger de 1 'entretien d'un troupeau de moutons, elle doit alors s'ouvrir de ses intentions au scppo. Ce dernier ira d'abord sur l'autel des ancêtres pour les avertir, mais cela ne suffit pas, il devra aussi réunir tous les sapra des autres lignages du village et c'est ensemble qu'ils consulteront les ancêtres. Si la réponse est favorable, le demandeur se verra autorisé à constituer (à ses frais...) un troupeau dont il ne sera jamais, bien sûr, que le gardien et au sein duquel le sapro puisera librement... mais élever des moutons est considéré comme une tâche noble et prestigieuse. Le premier bélier qui nấtra de la première brebis sera sacri- 
fié obligatoirement sur 1 'autel des ancêtres du lignage (borè), ensuite on laissera crốtre le troupeau.

De tout ce qui précéde, il ressort clairement qu'est codifié un rapport particulier entre l'espèce ovine et les ancêtres (borè) par chef de lignage sapro interposé.

- Les caprins.

Le bouc ou la chèvre sont très couramment sacrifiés, mais ils sont, à 1 'évidence, de qualité moindre aux yeux des Bobo que les moutons. Les caprins ne sont pas nécéssairement égorgés par un dignitaire sapro et chacun peut en élever à sa guise; seule condition posẻe au crëateur d'un troupeau : sacrifier le premier bouc né, non pas sur l'autel d'ancêtres borè, comme c'était le cas du premier bélier né, mais sur un autre autel, le saprè, où les ancêtres sont évoqués dans une perspective toute différente.

A la relation ovins - borè répond visiblement une relation caprins - saprè, nous y reviendrons dans la suite de cet article.

Ajoutons que les caprins, comme d'ailleurs les poulets et tous les autres animaux de sacrifice à 1 'exception des moutons, peuvent être librement vendus ou abattus pour servir de nourriture.

- Les bovins.

Les Bobo ne sont pas éleveurs de bovins, comme leurs voisins et coreligionnaires Bwa, il arrive cependant qu'ils sacrifient un taureau, acheté aux éleveurs peul qui parcourent. le pays.

Le seul autel qui reçoive de tels sacrifices est celui du village, kiri, et cela à l'occasion d'une cérémonie qui n'intervient que tous les sept ans.

- Les chiens.

Les sacrifices de chiens (beaucoup plus souvent des mâles que des femelles) ne sont pas rares chez les Bobo bien qu'ils soient loin d'égaler en fréquence ceux qu'on voit pratiquer chez leurs voisins Minyâka et Senufo-Nanerge; d'ailleurs, en 
plusieurs cas, les cultes où interviennent de tels sacrifices, chez les Bobo, sont en provenance de ces régions. Il n'en reste pas moins que 1'immolation de chiens est attestée dans le culte d'entités spirituelles typiquement bobo, celles, notamment, qui ont pour fonction d'assurer la défense du village $(d u b a)$ et de ses habitants ou de patronner les activités de chasse (nâko, dabu, wostni)... deux missions, on le remarquera, qui sont la vocation précisément du chien.

- Autres animaux.

La pintade domestique, dans un cas au moins (autel des jumeaux), peut remplacer le poulet.

Le chat (mâle ou femelle, mais toujours noir...) est sacrifié sur un autel de chasse et de guerre ( $d a b u$ ), c'est le seul cas que nous connaissions.

En dehors des animaux domestiques énumérés jusqu'ici et qui constituent la quasi totalité des victimes sacrificielles, il semble que sur quelques autels bien précis le sang d'animaux sauvages puisse être employé à des fins dont il n'est d'ailleurs pas assuré qu'elles soient toujours proprement "sacrificielles". Lorsque, par exemple, à l'occasion du rituel initiatique yele zo dâga (G.L.M., 1980, p. \$69) les membres de la classe d'âge kelebayelele font couler sur l'objet sacré polo le sang d'une petite antilope (ou, à défaut, d'une pintade sauvage) qu'ils s'étaient employés à capturer vivante en brousse, on peut parler sans doute de sacrifice. En revanche, lorsqu'un chasseur ayant tué un porc-épic ou un oryctérope ou encore une grande antilope enduit aussitôt de sang (1) le petit autel portatif dibi (de nâko) (2) qu'il porte sur lui, on ne saurait avec autant d'assurance qualifier de "sacrificiel" son acte.

(1). alors que le sang d'un animal domestique ne peut le toucher...

(2). Pour bâkoro et le dibi qui lui est associé, le sang de 1 'animal sauvage est même rapporté au village et rêpandu sur les autels intérieurs. 
b) - La mise à mort.

Si la.liste des victimes sacrificielles, avec tout l'éventail de leurs particularités physiques, offre un choix assez large aux Bobo et donc un cadre propice à 1 'élaboration d'un code, les méthodes employées pour mettre à mort ces victimes sont moins diversifiées et, bien que certaines d'entre ces méthodes soient spécifiquement réservées à des entités précises, il ne semble pas - au moins dans 1 'état actuel de nos connaissances - que le mode de mise à mort soit un critère suffisamment discriminant pour en infërer directement 1 'identité et surtout la nature profonde des très nombreux et divers destinataires sacrés. Nous nous bornerons donc ici à évoquer brièvement les méthodes qui ont cours.

Dans 1 'immense majorité des cas, les animaux sacrificiels sont simplement égorgês à l'aide d'un couteau et leur sang est répandu sur ou à côté de 1'autel. Bien qu'un certain nombre de variantes puissent êtrè observées d'une région à l'autre, on peut considérer 1a procédure suivante comme étant courante chez les Bobo.

Quand la victime est un poulet, le sacrificateur commence par introduire la lame de son couteau entre les deux parties du bec pour inciser la commissure et faire apparâ̂tre du sang. Le poulet étant aussitôt saisi par les pattes et placé tête en bas, des gouttes de sang coulent à terre. Le sacrificateur redresse ensuite le poulet et préléve de la main droite des plumes du dos et de la main gauche des plumes à la naissance de 1 a queue, ces plumes sont collées sur le sang versé. C'est alors que la gorge du poulet est tranchée d'un coup rapide de couteau. Le sacrificateur, prenant dans sa main droite l'animal qui se débat, fait en sorte que le sang coule, abondanment cette fois, sur les plumes posêes à terre (ou collées sur l'autel), puis il jette au sol le poulet, sur sa droite. Chacun observe les soubresauts de la victime et de la position qu'elle.prend dans la mort on conclut à l'acceptation (si le poulet est renversé sur le dos) ou au refus du don par le destinataire sacré.

Lorsque la victime est un quadrupède, on commence par couper quelques poils sur son front et sur sa queue, que 1 'on dépose sur le lieu adéquat, puis on égorge directement l'animal en aspergeant de sang 1 'autel ou ses abords. 
En dehors de ces mises à mort sanglantes avec égorgement existent d'autres techniques d'immolation, beaucoup plus rares, qui ont en commun le fait qu'elles ne sont accompagnées d'aucune effusion de sang.

C'est ainsi que la victime-(poulet ou quadrupède) peut être simplement assommée, en général d'un coup de kokolo (sorte de "canne" de fer, instrument cultuel de dwo - cf. G.L.M., 1980, p.112, fig. 3), et achevée, parfois, en étant projetée à terre avec violence. Ce mode de mise à mort semble être réservé aux victimes destinées à dwo (et tout particulièrement awosa) ou à des entités dérivêes de lui (sindo par exemple), ou encore être employé en des circonstances où des masques (donc dwo lui-même) sont amenés à agir (cas de funérailles spéciales). D'ailleurs, si 1 'opération est effectuée souvent par le sacrificateur, c'est parfois aussi le masque luimême (molo ou même kele) qui s'en charge. Notons qu'avec ce procédé, aucune goutte de sang n'est répandue; c'est d'ailleurs peut-être sa raison d'être puisque souvent, comme nous l'avons déjà signalê, dwo professe une certaine phobie des liquides, dont le sang.

Egalement en rapport avec dwo et le masque, la technique qui consiste à étouffer avec deux doigts un poussin et cela au dessus du nez d'un masque molo. Le poussin est ensuite jeté à terre où il achève de mourir. Ici encore aucun sang ne coule.

La victime peut être mise à mort de façon encore plus brutale, et, il faut le reconnâtre, plus cruelle.

Sur l'autel de l'entité tribale pô, tout particulièrement, mais aussi au grand cérémonial annuel birewa dâga, avec la présence et même la participation de masques de feuilles, on écartèle tout vif un poulet. Le poulet (noir au birewa dâga, d'espèce baba et de couleur rouge pour pô) est saisi par deux personnes, le sacrificateur et un assistant qui est souvent un forgeron ou parfois même un porteur de masque de feuilles. Chacun tient d'une main l'animal - l'un par la tête, I'autre par la queue - et tire violemment tandis que le sacrificateur tranche de son couteau au milieu du corps. La moitié antérieure est toujours jetée à I'ouest, la moitié postérieure à 1 'est. Sur pô parfois $1^{\prime}$ on ne fait même pas usage de couteau, chaque opérateur tient le poulet par une patte et une aile et tire de son côté... Les deux 
moitiés pantelantes sont également jetées 1 'une à 1 'ouest, 1 'autre à 1'est. Les restes de l'animal ainsi supplicié - dont le sang, remarquons-le à nouveau, n'a pas touché 1 'autel - ne peuvent être approchés et consommés que par un forgeron car cette mise à mort est généralement associée à un rite d'éviction du mal et la vitime est jugée comme étant dangereusement polluée (1).

Le comble de la cruauté (à nos yeux, certes, mais aussi pour les Bobo, qui en conviennent) est atteint avec la torture infligée à des animaux lors du rituel tî na timi daga qui clôt l'enterrement solennel syèbt. A cette occasion, en effet, on casse une patte antérieure et une patte postérieure d'un mouton et d'une chèvre et on les laisse agoniser dans leurs souffrances toute la nuit. Nul sang n'est versé, mais ces animaux, qui sont en fait des viatiques pour le dêfunt, sont-ils à proprement parler "sacrifiés" et ne sont-ils pas plutôt 1 'objet d'un simple "traitement rituel" ?

A l'opposé de ces mêthodes violentes, se présente un dernier cas, de tous sans doute le plus spectaculaire et qui reste tout à fait énigmatique : celui de la mise à mort d'un animal sans intervention humaine décelable. Pour quelques très rares entitês patronnes de la chasse (dabu et dibi de nâko notamment), la victime (chien, chèvre, chat ou poulet, selon le cas), dont les pattes sont tenues par deux assistants, est placée la poitrine contre l'autel. Le sacrificateur, sans toucher à aucun moment à l'animal, s'approche, prononce une courte prière et... attend. La mort est en principe foudroyante. Le sang ne doit pas apparâtre, on inspecte d'ailleurs 1a bouche de 1'animal à cet effet; si des tracés de sang étaient visibies, le sacrifice serait considéré comme étant refusé.

Ayant longuement énumêré les espèces animales sacrifiables et décrit les techniques d'immolation, reprenons le cours des opérations au point où nous 1 'avons interrompu.

(1). Cf. Le Moal, G., "Rites de purification et d'expiation" in Systèmes de Signes. Textes réunis en hommage à G. Dieterlen. Paris Hermann. 1978. 
Avec la mise à mort des victimes principales - part personnelle avons-nous dit de 1 'entité spirituelle destinataire - s'achève la phase incontestablement majeure du procès sacrificiel. Dès lors, en effet, le contrat est rempli - si, du moins, le destinataire sacré a clairement accepté les dons de vies animales car en cas contraire tout serait remis en cause.

A ce moment, l'atmosphère, jusqu'alors tendue, voire fébrile, devient joyeuse et bruyante. Pour autant, l'action n'est point achevêe : dès après que le quadrupède a été immolé, on entre en effet dans une nouvelle phase de libations et/ou d'offrandes qui s'accompagnera d'un ultime sacrifice sanglant.

\section{Offrandes et libations.}

Si l'entité à laquelle est dédié le sacrifice est de celles qui demandent de la pâte de mil sêkèzè, le moment est venu de lui en faire $1^{\prime}$ offrande. Rappelons que l'eau qui surnageait au dessus de cette pâte a fait l'objet d'une libation tout au début de la cêrémonie. Le sacrificateur prend donc un morceau de cette pâte, le délaye dans un peu d'eau et verse la bouillie obtenue sur l'autel. Aussitôt après il prélève un autre morceau de sêkèzè, mais le laisse en l'etat pourle coller derechef sur l'autel.

Bien entendu, dans le cas ou fogo zo était requis, il n'en est point fait usage à cet instant puisqu'il a été entièrement versé lors des libations prêliminaires; on passe donc directement à la suite.

Après le dépôt de sêkèzè, on procède à la dernière immolation: un poulet, encore et simplement "pour la bonne santé" de toutes les personnes présentes.

On peut alors en revenir à la bière de mil dont seules quelques gouttes avaient été versées auparavant, au moment des libations. A nouveau l'autel reçoit donc un peu de cette boisson fermentée qu'aiment tout autant les Dieux que les hommes tandis que le prêtre prononce quelques ultimes paroles reconnaissantes.

Les jarres de bière, quasiment pleines encore, sont une invite à entrer sans plus tarder dans la phase finale du cérêmonial. 
7. Consommation.

A quelque distance de 1 'autel on plume les poulets sacrifiés et 1 'on prépare un maigre feu pour les griller sommairement. Pendant ce temps commence la distribution de la bière, acte riche d'enseignements pour 1 'observateur car, surtout si 1 'audience est nombreuse et diversifiée (cas d'un sacrifice intéressant tout le lignage ou plusieurs lignages du même clan ou encore de sacrifices faits à 1'échelle du village dans son entier, voire de plusieurs villages), l'ordre dans lequel les parts sont donnēes ainsi que l'identité des bénéficiaires sont strictement prédéterminès et donc révélateurs des hiérarchies qui sous-tendent tout le système social et religieux.

Plus important encore aux yeux des participants et plus instructif aussi pour l'observateur est le partage des viandes sacrificie1les grillées qui suit la distribution de bière car entrent êgalement en ligne de compte la nature et la qualité (essentiellement symbolique) des morceaux découpés.

Les diverses parties du corps des poulets et, de façon encore plus significative, des quadrupèdes sont attribuées avec précision, de telle sorte qu'en le corps divisé de la victime on peut voir comme une projection métaphorique du système multilignager communautaire en activité dans tout village bobo et qui se caractêrise par des échanges contractuels visant à créer un état de symbiose (G.L.M., 1980 , p. 66). A chaque sacrifice - chez les Bobo come dans bien d'autres populations africaines où cela a déjà étẻ mis en lumière - sont donc associés des actes consacrés à la réaffirmation du contrat social et peut-être même à sa réactualisation. Nous disons bien "associés" car ces actes - pour importants qu'ils soient - ne nous semblent pas être chez les Bobo du moins - la fin première du procès sacrificie1. Pour cette raison - qu'il nous faudrait longuement justifier - et pour ne pas sortir du cadre du présent article, nous ne nous appesantirons pas sur les modalités fort complexes du partage des viandes sacrificielles, quitte à revenir un jour sur ce sujet. Remarquons pourtant au passage que, chez les Bobo, aucune part ne revient à l'entité destinataire du sacrifice. La part des entités spirituelles, ce sont seulement le sang et les plumes, ainsi que les libations et les parcelles de nourritures oblatives déposées sur l'autel. 
C'est sur place, autour de l'autel, que seront consommées 1a bière et la chair des poulets. Cela se fait sans cérémonie et dans un joyeux brouhaha. Pourtant il s'agit là d'un acte essentiel, le second en importance'après l'immolation elle-même pensons-nous, et dont le sens, si nous étions en mesure de le percer, éclairerait sans doute de façon décisive la problématique bobo du sacrifice. Faute d'une pénétration suffisante de la pensée bobo en ce domaine, nous ne nous hasarderons pas à proposer des significations et nous resterons très prudents quant à l'emploi des termes qui reviennent si souvent dans les textes à ce propos - celui de "communion" notamment. Un principe seulement sera évoqué ici car il donne la mesure de 1'importance que revêt, pour le Bobo, l'absorption rituelle des viandes sacrificielles : consommer, pense-t-on en effet, plonge ipso facto l'individu dans un état de pleine participation à 1 'acte sacrificiel. Manger la chair des victimes n'est donc pas sans effets : pour le sacrifiant, cela rentre bien sûr dans la logique de son entreprise - d'une certaine façon d'ailleurs, consommer c'est parapher 1 'accord que 1 'entité spirituelle vient de donner en acceptant les vies offertes; pour les autres participants, sacrificateur compris, se nourrir de cette chair c'est être associé possiblement aux bénéfices de l'opération (rappelons que plusieurs de ces poulets qu'on consomme ont été tués pour "la bonne santé" de tous), mais c'est aussi s'exposer à des suites malheureuses qui frapperont collectivement si quelque erreur liturgique a étê commise, par exemple, ou si... chose bien courante, le sacrifiant ne remplit pas toutes les promesses qu'il a faites à l'entité destinataire dans le cadre du sacrifice. En fin de compte, immoler est de beaucoup moins de conséquence que consommer. Cela est si vrai que, dans un cas de nécessité notamment, un individu peut outrepasser sans danger l'interdit qui le frappe de faire couler le sang sur un autel donné pourvu qu'il s'abstienne de manger la viande partagée. "On peut tuer un animal, si on ne mange pas sa chair, c'est comme si $1^{\prime}$ 'on n'avait rien fait" nous disait un forgeron qui avait étê amené à sacrifier sur kwere, culte privé de la Foudre, duquel pourtant tous les forgerons sont exclus (G.L.M., 1980, p. 133).

Le partage et la consommation immédiate de la bière et des poulets 
immolés ne marquent la fin du procès sacrificiel que dans le cas où l'entité spirituelle concernée ne demande, en matière de libation que de la farine délayée fogo zo. En revanche, si l'emploi de sêkèzè est prescrit, il reste à organiser ce repas collectif dont nous avons parlé plus haut et au cours duquel seront partagés - toujours en suivant les règles d'attribution édictées - non seulement les quantités souvent importantes de bière réservées pour ce moment et aussi quelques poulets restants, mais surtout le ou les quadrupèdes sacrifiés. (1) qu'accompagnera enfin la pâte de mil sêkèzè préparée.

Dans le cours de notre description des phases du procès sacrificiel, nous avons êté amenẻ à faire êtat des choix qui s'exercent, à différentes reprises, tant en ce qui concerne les nourritures oblatives et les libations que les victimes animales. Cela n'est en rien particulier aux Bobo : l'existence d'une liste limitative de denrées ou animaux susceptibles d'être employés au cours des rites du sacrifice est attestée dans toutes les sociêtês. Faisant lui-même cette remarque et s'interrogeant sur les possibilités qui pourraient s'offrir de comprendre les raisons de ces choix, Michel Cartry rappelait fort opportunément, dans le premier cahier de notre revue consacré au sacrifice (2), que "Tout ordre social est fondé sur un ensemble de systèmes classificatoires primaires (3) dont il est impossible de retracer complètement la genèse". La tentation d'élucider les raisons qui ont présidé aux choix d'êtres et d'objets propres à chaque socié-

(1). Les quadrupèdes immolés dans le cadre d'un sacrifice avec fogo zo sont partagés sur place mais les morceaux sont emportés par chacun et consommés en famille à domicile.

(2). Cartry, M. , p. 153.

(3). C'est nous qui soulignons. 
té est donc vaine. A coup sûr, il n'est plus guère possible aujourd'hui d'expliquer pourquoi les Bobo ont privilégié telle espèce animale plutôt que telle autre pour en faire des victimes sacrificielles ou encore pourquoi le choix a été fait des seules couleurs blanc, rouge et noir.

Certes, des éléments de réponse sont parfois présents dans l'appareil symbolique, mais celui-ci ne rend pas pleinement compte de la spécificité bobo, par le fait qu'il est, pour une bonne part, le fruit d'hêritages transculturels. D'autres justifications existent (fondées souvent sur des contes ou légendes), mais qui sont trop obligeamment offertes pour ne pas être suspectes : elles n'ont, en général, été conçues qu'a posteriori.

Ce qui, en revanche, peut être d'un plus grand intérêt, c'est, d'une part, la façon dont sont traités les élêments des systèmes primaires de classification - les hiérarchies introduites, donc, ou les échelles de valeur assignées - et ce sont d'autre part, les articulations établies entre ces systèmes et les grandes divisions de l'ordre social et/ou religieux. Ces divers traitements et jeux de correspondances mènent en général à l'établissement de vêritables codes dans lesquels on peut voir, cette fois, la marque personnelle d'une culture donnée; 1'expression originale de son idéologie a en effet toute chance d'être inscrite dans ces combinatoires souvent très savantes qui sont en fait des registres de représentations intellectuelles exprimées en termes métaphoriques et liées entre elles par des associations qui sont en elles-mêmes porteuses de sens.

Quand de tels codes existent dans le cadre d'une institution, quand ils sont de référence constante et quand leur complexité laisse augurer une iritéressante richessse de contenu, alors un déchiffrement s'impose.

C'est ce que nous avons tenté de faire chez les. Bobo où nous avons étudié le code régissant les rapports entre la nature des victimes ou offrandes sacrificielles et la qualité des entités spirituelles destinataires.

L'exposé des résultats de ce travail fera 1 'objet de la deuxième partie de notre article. 


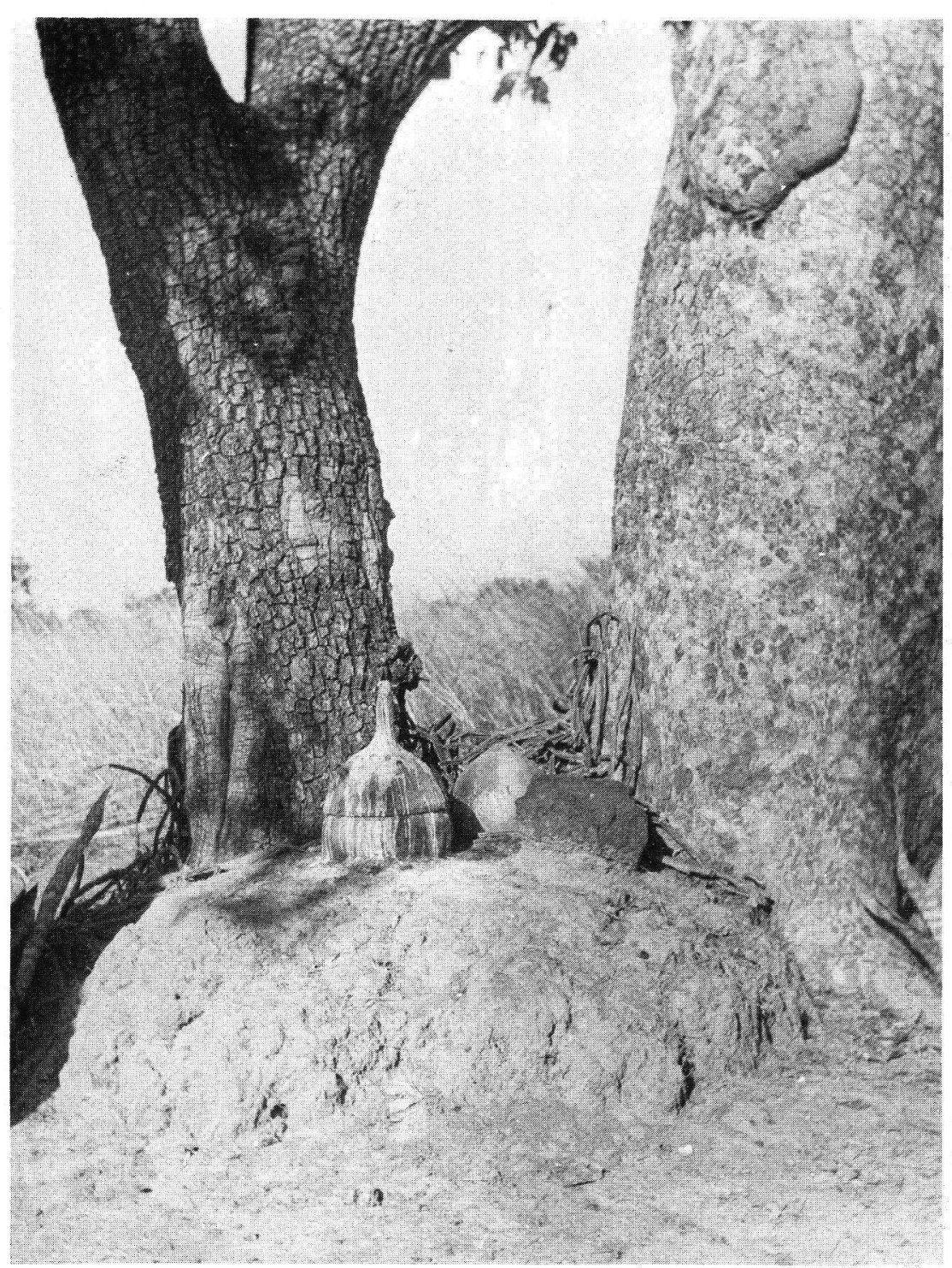

Photo 1

Autel de nâko, entité spirituelle protectrice des chasseurs 


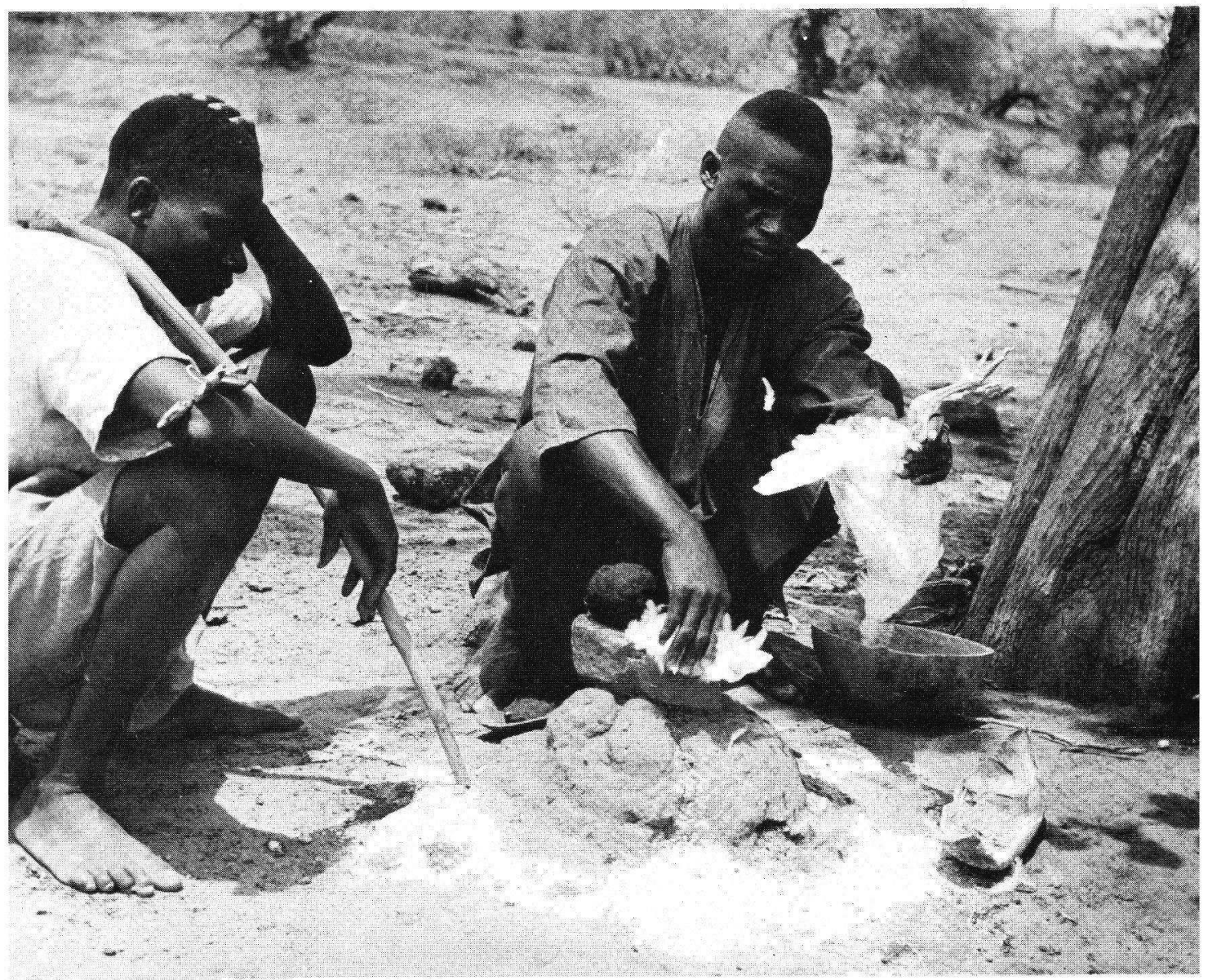

Photo 2

Immolation d'un poulet. Remarquez la

cendre répandue autour de l'autel 